\title{
Expression of the Wnt antagonist Dickkopf-1 in endometriosis
}

\author{
Mariz Kasoha $^{1 *}$, Laura Golbach ${ }^{1}$, Panagiotis Sklavounos ${ }^{1}$, Sebastian Findeklee ${ }^{1}$, Ingolf Juhasz-Böss ${ }^{1}$, Hermann Eichler ${ }^{2}$ and Erich-Franz \\ Solomayer ${ }^{1}$ \\ ${ }^{1}$ Department of Gynecology, Obstetrics and Reproductive Medicine, University Medical School of Saarland, 66421 Homburg / Saar, Germany \\ ${ }^{2}$ Institute of Clinical Hemostaseology and Transfusion Medicine, University Medical School of Saarland, 66421 Homburg / Saar, Germany
}

\begin{abstract}
Background: Dickkopf-1 protein (Dkk1), a major inhibitor of Wnt/ $\beta$-catenin signaling, is a secretory glycoprotein that has been found to be involved in the pathogenesis of several human diseases and to provide a promising diagnostic and therapeutic tool in diverse cancer entities. Endometriosis shares some characteristics with invasive tumors. The present study aimed to investigate whether Dkk1 expression is dysregulated in endometriosis.

Methods: 188 subjects including 109 women with histologically confirmed endometriosis and 79 healthy women were recruited. Serum Dkk1 levels were measured by ELISA in 97 and 75 women with- and without endometriosis respectively. Gene expression levels of Dkk1- and $\beta$-catenin were assessed by quantitative polymerase chain reaction in 28 different human tissue types, including 4 normal endometrium tissues, 8 normal peritoneal tissues, and 16 endometriosis tissues.

Results: Endometriosis patient women showed significantly higher serum levels of Dkk1 compared with healthy women [2999 pg/ml (947-5104) vs $2216 \mathrm{pg} / \mathrm{ml}$ $(1008-4109)$ respectively; $p<0.0001]$. No significant differences in Dkk1 gene expression levels were found between the different tissue types but it showed, within endometriosis tissue group, to be upregulated in cases with more severe disease. $\beta$-catenin gene expression was found to be down regulated in endometriosis tissues compared with normal endometrium tissues.

Conclusions: To the best of our knowledge, this is the first study to investigate serum levels of Dkk1 in patients with endometriosis. Our results demonstrated aberrant expression of two main components of $\mathrm{Wnt} / \beta$-catenin signaling in endometriosis. Interventions in this signaling transduction pathway can contribute to development of new targets for disease control and therapy. Dkk1 could serve as diagnostic biomarker in endometriosis. However, further multicentre large-scaled studies are required.
\end{abstract}

\section{Introduction}

Wnt $/ \beta$-catenin signaling pathway is mediated by the tight regulation of $\beta$-catenin stability and widely known to be involved in a wide range of physiologic and pathologic processes throughout the body. In the presence of Wnt, signaling is kept on by binding of Wnt proteins to the N-terminal extra-cellular cysteine-rich domain of a Frizzled (Fz) family receptor and other co-receptors such as lipoprotein receptor-related protein (LRP)-5/6. This binding initiates a signaling cascade that inhibits the so called "destruction complex" which targets $\beta$-catenin for ubiquitin-mediated proteasomal degradation. Consequently, free cytoplasmic non phosphorylated $\beta$-catenin accumulates and relocates into the nucleus, where it binds to different transcription factors promoting transcription of a broad spectrum of Wnt downstream target genes including those that promote cell proliferation and survival. In the absence of Wnt, the destruction complex induces phosphorylation of $\beta$-catenin resulting in its degradation [1].

In the endometrium, Wnt $/ \beta$-catenin signaling pathway serves a decisive role in endometrial gland formation and mesenchymal development and involves in the adhesion, invasion and angiogenesis of the ectopic endometrium [2]. Sanchez and colleagues showed that the expression of specific members of Wnt pathway and its pivot molecule $\beta$-catenin were dysregulated in luteinized granulosa cells derived from endometriosis patients compared with those from control women. This aberrant expression was characterised by an increased apoptosis suggesting that $\mathrm{Wnt} / \beta$-catenin signaling could be involved in causing granulosa cell atresia [3]. Furthermore, it has been showed that $\mathrm{Wnt} / \beta$-catenin signaling trigger fibrogenesis associated with endometriosis via stimulating fibrotic markers expression of and these effects were prevented by treatment with small-molecule antagonists of certain components of this signaling pathway [4]. Zhang et al. showed that stimulation of human endometrial stroma cells (HESCs) with E2 increased mRNA expression of vascular endothelial growth factor (VEGF) and this effect can be prevented when cells were transfected with $\beta$-catenin si-RNA suggesting the plausible role of $\mathrm{Wnt} / \beta$-catenin signaling in initiating endometriosis via angiogenesis stimulation [5].

Dickkopf (Dkk) proteins, exemplified by Dkk1, are LRP5/6 ligands antagonists and considered potent inhibitors for Wnt $/ \beta$-catenin signaling [6]. Overexpression of Dkk1 is associated with several

*Correspondence to: Mariz Kasoha, Department of Gynecology, Obstetrics and Reproductive Medicine, University Medical School of Saarland, 66421 Homburg / Saar, Germany, Tel: +49 (0) 6841/16-28199; Fax: +49 (0) 6841/1628110; E-mail: mariz.kasoha@uks.eu

Key words: Wnt/ $\beta$-catenin signaling, dickkopf-1 (Dkk1), endometriosis, biomarker, pathogenicity

Received: July 30, 2019; Accepted: August 23, 2019; Published: August 27, 2019 
diseases including various types of cancers [7] and anti-Dkk1 antibodies are being tested experimentally and clinically as potential therapy agents in different bone diseases and cancers and preliminary results demonstrated a favourable safety profile introducing promising therapy options $[8,9]$. We have previously reported that protein levels of Dkk1 in peripheral blood are increased in breast cancer patients compared with control women. In addition, increased protein expression of Dkk1 in breast cancer cells and cancer surrounding tissues has been documented [10]. Moreover, elevated blood levels of Dkk1 were found to be positively correlated with poor prognosis in lung cancer and cervical cancer $[11,12]$. Therefore, blood levels of Dkk1 could be used as non-invasive diagnostic- and prognostic biomarker in certain cancer entities.

Diagnosis of endometriosis in many affected women is often delayed resulting in reduced quality of life and decline in reproductive potential and fertility imposing a significant economic burden $[13,14]$. Although direct laparoscopic visualization and histologic confirmation remains the gold standard for diagnosing endometriosis, a non-invasive tool could facilitate earlier diagnosis and intervention that could ultimately improve disease consequences. Blood level of Dkk1 is considered as an advantageous biomarker because it is noninvasive, has a simple detection protocol, and is inexpensive to quantify. Our present study aimed to investigate whether blood protein levels of Dkk1 and/or its tissue gene expression are dysregulated in women with endometriosis compared with healthy women.

\section{Material and methods}

\section{Study participants and sampling}

Our study included 188 women consisting of 79 healthy women and 109 endometriosis patient women. Details on the study case series are illustrated in Figure 1. Patient cohorts were recruited from women admitted to our department with signs of endometriosis from May 2013 and December 2016. Inclusion criteria were histologically confirmed endometriosis and no recent pathological finding in uterus cavity. Serum samples were obtained from 97 patient women. Patients' characteristics are outlined in Table 1 . The median (Range) age for the patients at the time of the sample drawn was 34 years (16-52). Disease staging was determined according to the revised American Society for Reproductive Medicine (rASRM) [15]. Serum of 75 age-matched healthy women [30 years (19-52)] was also collected. These healthy cohorts were recruited from those who had undergone comprehensive medical screening prior to blood donation at the Institute of Transfusion Medicine of our university hospital. All serum samples were frozen in aliquots at $-80^{\circ} \mathrm{C}$ until being analysed.

Tissue samples were collected from 22 women with endometriosis during laparoscopic surgery. Specimens included 16 endometriosis tissues and 8 normal peritoneal tissues of which 2 matched normal peritoneal- and endometriosis tissues were collected from the same patient. Furthermore, normal endometrium tissue samples were collected from 4 women who underwent laparoscopic surgery for benign indications other than endometriosis. Endometrial pathology was excluded in healthy controls. Tissues were directly flash-frozen in liquid nitrogen and immediately transferred to $-80^{\circ} \mathrm{C}$ until used.

All study participants were documented not to be pregnant and have no history of malignancies.

This study was approved by the local Ethic Committee of the Medical Association of the Saarland (Reference number: 23/16). Human blood samples and tissue samples were collected from all individuals after signing a written and informed consent that is approved by the Ethic Committee.

\section{Serum analysis}

Serum protein levels of Dkk1 were measured by Enzyme-Linked Immunosorbent Assay (ELISA) in 75 healthy women and in 97 patient women using Quantikine immunoassay control set 921 for human Dkk1 (QC241) and Human Dkk-1 Quantikine ELISA Kit (DKK100B) from

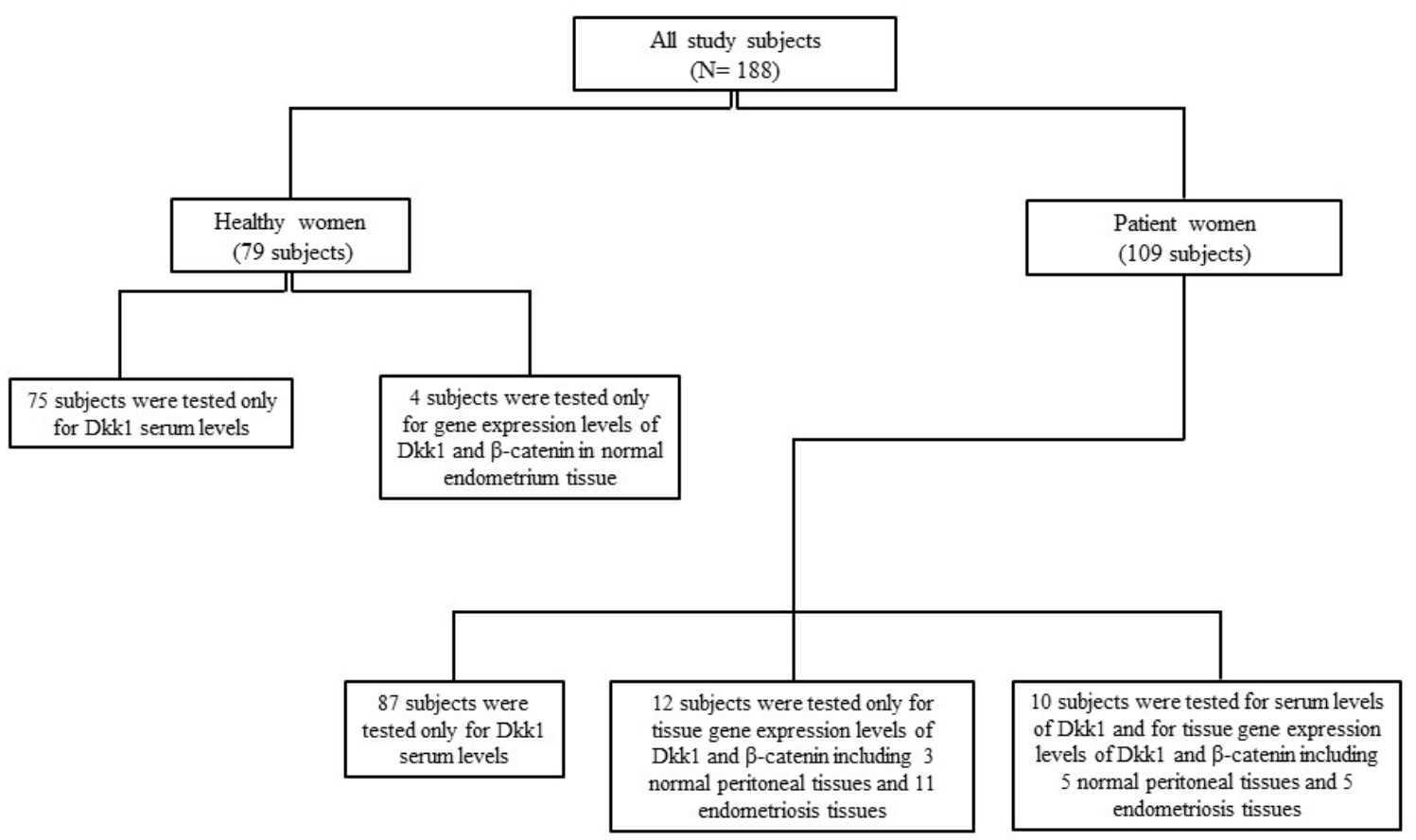

Figure 1. Details on the study case series 
Table 1. Characteristics of patients in whom Dkk1 levels were tested in serum

\begin{tabular}{|l|c|c|}
\hline & Number of patients & $\%$ \\
\hline All patients & 97 & $100 \%$ \\
Age: Median (Range)years & $34(16-52)$ & \\
\hline Disease occurrence & & 55.7 \\
Patients with first time & 54 & 30.9 \\
diagnosis & 30 & 13.4 \\
Patients with one recurrence & 13 & \\
Patients more than one & & 85.6 \\
recurrence & $83 \ddagger$ & 14.4 \\
\hline Disease classification $\dagger$ & 14 & \\
Not deep infiltrated & & \\
Deep infiltrated & & \\
\hline
\end{tabular}

†Cases were according to the revised American Society for Reproductive Medicine (rASRM). $\$$ Included 47 cases with minimal-, 17 cases with mild-, and 19 cases with moderate endometriosis.

R\&D Systems ${ }^{\oplus}$ (Germany) according to manufacturer's instructions. Optical density was measured using 96-well microplate reader (SunriseTecan, Life Science). Dkk-1 concentrations were obtained with a fourparameter logistic curve fitted against a standard curve and multiplied by the dilution factor using Magellan 7.2 Ink Data Analysis Software (Life Science-Tecan). All measurements were performed in duplicate.

\section{Tissue analysis}

Histologic test: Cryosections were developed from obtained tissue samples and consequently stained according to the Hematoxylin and eosins (H\&E) protocol for frozen tissue. Then, stained sections were analyzed under Zeiss microscope (Axioskop 40, Carl Zeiss, Germany) and selected pictures were captured with attached digital camera (AxioCam MRC, Carl Zeiss, Germany) using Axiovision Documentation Rel.4.8 program. Histological evaluation was done by an experienced pathologist from the department of Pathology at our university hospital. Histologic diagnosis of endometriosis was confirmed by the presence of endometrial glands and stroma with or without haemosiderin-laden macrophages. All tested peritoneal tissues showed negative diagnosis for endometriosis (Figure 2).

RNA extraction and cDNA synthesis: Levels of Dkk1- and $\beta$-catenin genes were analyzed in 4 normal human endometrium tissues, 8 normal peritoneal tissues, and 16 endometriosis lesions. A maximum amount of 15-20 mg frozen tissues were distributed and homogenized in $300 \mu \mathrm{l}$ of RNeasy lysis buffer (Qiagen, Valencia, CA, USA) using TissueLyser LT Adapter and stainless steel beads from Qiagen. RNA extraction was performed using RNeasy MiniKit (Qiagen, Valencia, CA, USA) following the manufacturer's instructions. Thereafter, Ambion* TURBO DNA-free ${ }^{\text {Tx }}$ DNase kit (Life Technologies GmbH, Darmstadt, Germany) was used to remove contaminating DNA from RNA preparations, and to subsequently remove the DNase and divalent cations from the sample. Concentration and purity of extracted RNA were measured using Thermo Scientific $c^{\text {Tx }}$ NanoDrop 2000. In addition, further detecting of RNA integrity was done using Agilent RNA 6000 Nano Reagents Part I (Agilent Technologies, Waldbronn, Germany) and Bioanalyser Agilent 2100 from Agilent Technologies. Afterward, reverse transcribed complementary DNA (cDNA) was synthesized with the High Capacity cDNA Reverse Transcription Kit as described by the manufacturer (Applied Biosystem, Foster City, CA, USA).

Real Time RT-PCR: The PCR efficiency was tested to confirm that the results will not be influenced by amplification of contaminating DNA in the RNA samples and was found to be in a range between $90 \%$ and $100 \%$ for tested Dkk1- and $\beta$-catenin genes. Glyceraldehyde-3phosphate dehydrogenase gene $(G A P D H)$ was used as a housekeeping gene. Quantitative real-time PCR was performed in triplicates with dilutions of 10 ng cDNA using TaqMan Gene Expression Assays by Life Technologies (Table 2) on an Applied Biosystems 7500 Fast RealTime PCR System. All samples with a cycle threshold $\left(C_{t}\right.$ coefficient of variation value $>5 \%$ were retested. In addition, a no template control (NTC) was included in each run, and the resulting $C_{t}$ values were normalized to GAPDH mRNA expression. The RT-PCR data were analyzed using the 7500 Software v.2.0.5 (Life Technologies GmbH, Darmstadt, Germany).

Statistical analysis: All statistical tests were performed at a twosided comparison-wise significance level of 5\%. Continuous variables were analysed descriptively using mean, standard deviation, median and range of the observations. Categorical variables were analysed descriptively using absolute and relative frequencies. The null hypothesis was tested using two tails Student's t-test and Mann-Whitney $U$ test/ or Kruskal-Wallis-Test for parametric and non-parametric continuous data respectively. All data obtained from this study were analyzed using SPSS version 21 (IBM, Armonk, NY, USA).

\section{Results}

\section{Serum levels of Dkk1 are increased in endometriosis patients}

Serum levels of Dkk1 were measured in 97 endometriosis patient women patients [Age: Mean (range): 34 (16-52) years] and in 75 agematched healthy women [30 years (19-52)]. Our results revealed that endometriosis patients had significantly higher serum levels of Dkk1 compared with healthy women [Mean (Range): 2999 pg/ml (947-5104) vs $2216 \mathrm{pg} / \mathrm{ml}$ (1008-4109) respectively; $p<0.0001$ ] (Figure 3).

Then, we have compared serum levels of Dkk1 between different subgroups according to disease occurrence and disease classification and no significant differences were reported (Table 3 ).

Gene expression levels of Dkk1 and $\beta$-catenin in normal endometrium tissues, in normal peritoneal tissues, and in endometriosis tissues

For this purpose, we have collected endometrium tissues from 4 women (aged 26, 44, 44, and 46 years) who underwent laparoscopic surgery for benign indications other than endometriosis. In addition, we collected normal peritoneal tissues and endometriosis tissues from 22 patient women. The final tissues that were valid to be used for

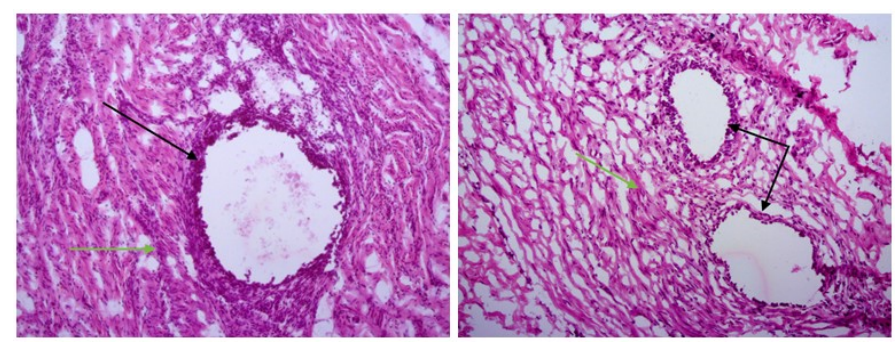

Figure 2. Hematoxylin and eosins (H\&E) staining of cryosections of endometriosis tissues. Histologic diagnosis of endometriosis was confirmed by the presence of endometria glands (Black arrows) and stroma (Green arrows) with or without haemosiderin-laden macrophages

Table 2. TaqMan ${ }^{\circledR}$ Gene expression assays used for quantitative RT-PCR

\begin{tabular}{|c|c|}
\hline Gene & Taqman assay ID \\
\hline$d k k 1$ & Hs00183740_m1 \\
\hline$C T N N B 1(\beta$-catenin $)$ & Hs00355045_m1 \\
\hline GAPDH ${ }^{\mathrm{a}}$ & Hs03929097_m1 \\
\hline
\end{tabular}

${ }^{a}$ Endogenous gene 
real time PCR test included 16 endometriosis tissues and 8 normal peritoneal tissues, of which 2 matched normal peritoneal- and endometriosis tissues were available. The rest samples were excluded from the study because of the low purity and integrity of the extracted RNA. Characteristics of patients in whom gene expression tests were performed are listed in Table 4.

Our RT-PCR results showed no significant difference in $d k k 1$ expression levels within the three tested tissue groups (Figure $4 \mathrm{~A}$ ). However, in the endometriosis tissue group, we found that $d k k 1$ expression level in 5 deep infiltrated endometriosis tissues was significantly higher than it in 11 not deep infiltrated endometriosis tissues [Mean (Range) $\Delta C_{t}: 3.4 \pm 2.8$ vs $8.32 \pm 4.16$ respectively; $p=0.032$ ] (Figure 4B). Expression levels of $d k k 1$ did not differ according to disease occurrence status.

On the other hand, we found that $\beta$-catenin expression levels in endometriosis tissues was downregulated compared with normal endometrium tissues (Figure 5). No other significant differences in $\beta$-catenin expression levels were reported between other tissue groups. In addition, $\beta$-catenin expression levels in endometriosis tissues showed no significant alteration according to any of patient's specifications including disease occurrence status and disease severity (data are not shown).

\section{Discussion}

Endometriosis is considered as a multifactorial disease affected by immunological, hormonal, and environmental factors. However, the Sampson's retrograde hypothesis remains the most widely accepted theory, of which a main part implicates the epithelial to mesenchymal transition (EMT) as the pathogenic mechanism $[16,17]$. Wnt/ $\beta$-catenin signaling is considered essential in EMT activation and regulation [18]. As aforementioned, aberrant $\mathrm{Wnt} / \beta$-catenin signaling has been linked to the pathogenesis of endometriosis via various mechanisms [3-5].

Table 3. Serum levels of Dkk1 (pg/ml) in different patients subgroups

\begin{tabular}{|c|c|c|c|c|c|}
\hline Characteristic & Subgroup 1 & Subgroup 2 & Subgroup 3 & Subgroup 4 & $p$ \\
\hline Disease occurrence & $\begin{array}{l}\text { Patients with first time diagnosis } \\
3031(1408-4579)\end{array}$ & $\begin{array}{l}\text { Patients with one recurrence } \\
2889(1008-5104)\end{array}$ & \begin{tabular}{|c|} 
Patients more than one recurrence \\
$3034(974-4214)$
\end{tabular} & - & NS \\
\hline Disease classification & $\begin{array}{c}\text { deep infiltrated } \\
2811(1678-3820)\end{array}$ & $\begin{array}{c}\text { Minimal } \\
3279(1408-5104)\end{array}$ & $\begin{array}{c}\text { Mild } \\
2848(1953-3647)\end{array}$ & $\begin{array}{c}\text { Moderate } \\
3145(947-4597)\end{array}$ & NS \\
\hline
\end{tabular}

Results are showed as [Median (Range)]. $P$ value is tested with Kruskal-Wallis-Test. NS: not significant.

Table 4. Characteristics of patients in whom Dkk1- and ß-catenin gene expression tests were done

\begin{tabular}{|l|c|c|}
\hline \multicolumn{1}{|c|}{ Number of patients } \\
\hline All patients & 22 \\
Age: Median (Range)years & $35(24-46)$ \\
\hline Disease occurrence & \\
Patients with first time diagnosis & 9 \\
Patients with one recurrence & 7 \\
Patients more than one recurrence & 6 \\
\hline Disease classification† & \\
Not deep infiltrated & $16 \%$ \\
Deep infiltrated & 6 \\
\hline
\end{tabular}

$\dagger$ Cases were classified according to the revised American Society for Reproductive Medicine (rASRM). $\$$ Included 7 cases with minimal-, 3 cases with mild-, and 6 cases with moderate endometriosis.

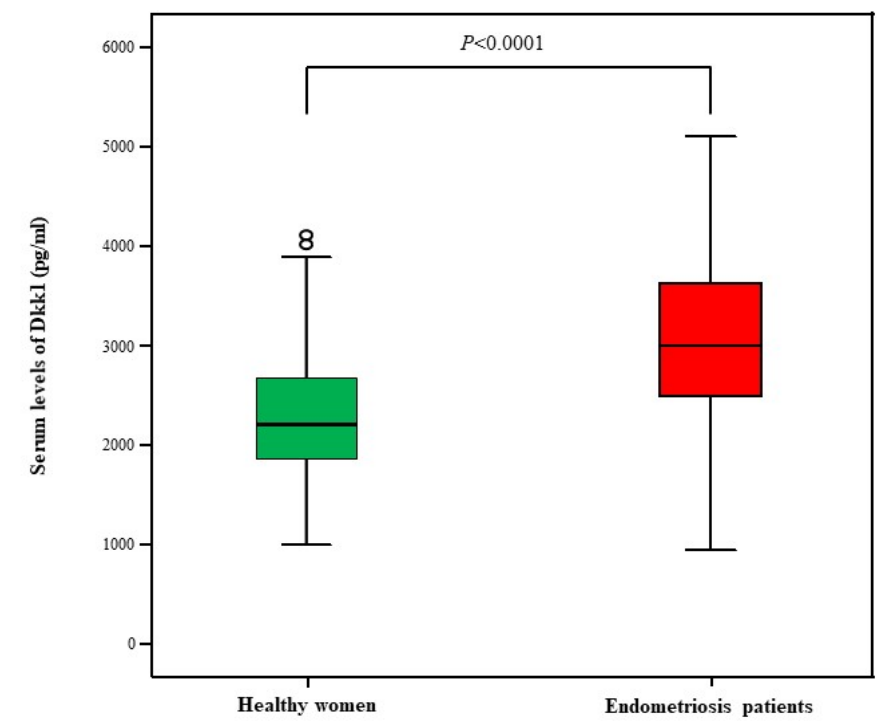

Figure 3. Serum levels of Dkk1 (pg/ml) in healthy women and in endometriosis patients. Dkk1serum levels in patients [2999 pg/ml (947-5104)] were significantly higher than those in controls $[2216 \mathrm{pg} / \mathrm{ml}(1008-4109)](p<0.0001)]$ 

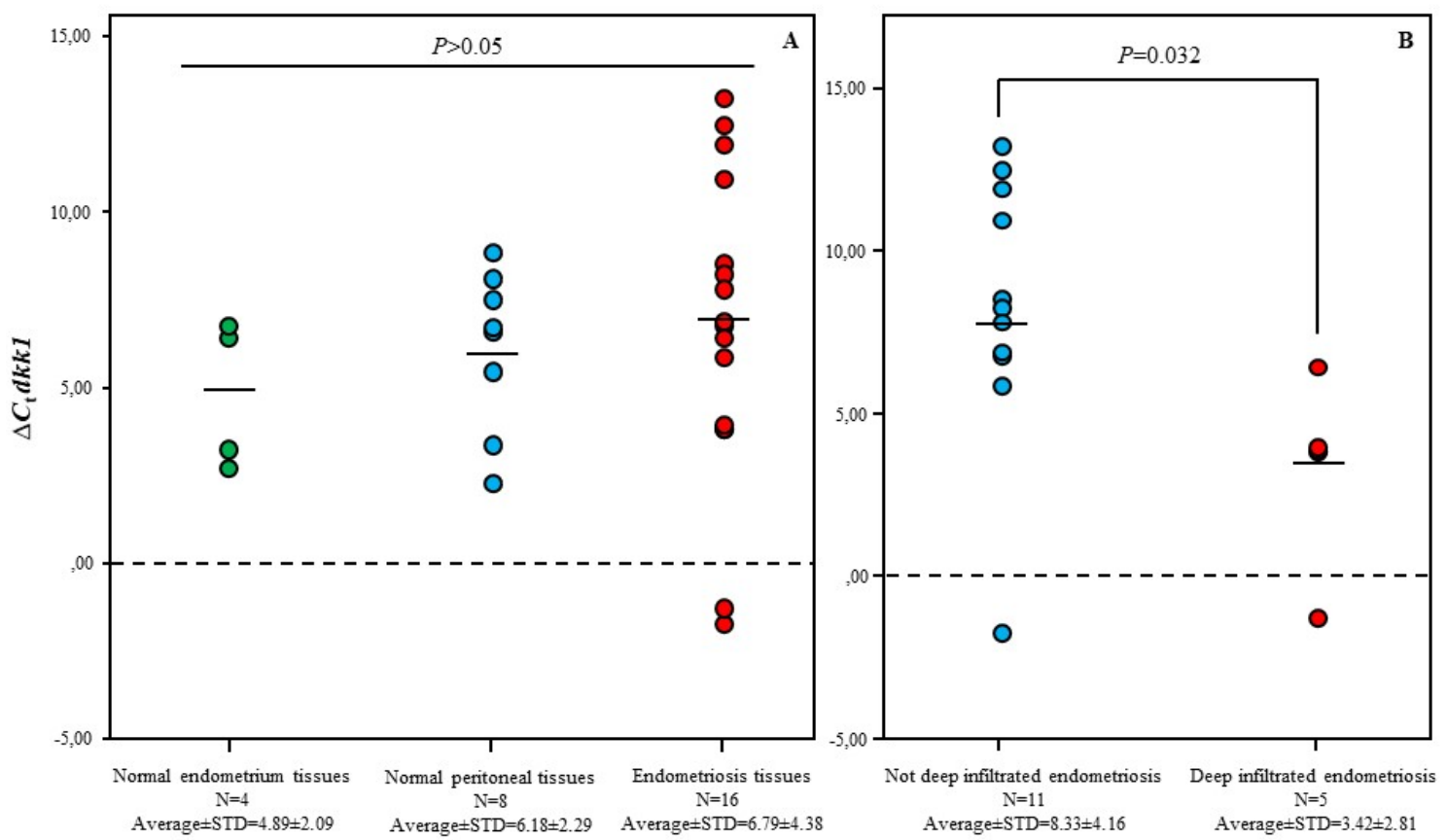

Figure 4. Gene expression levels of Dkk1 in different tissue samples. A: Differences in gene expression levels of Dkk1 in normal endometrium-, in normal peritoneal- and in endometriosis tissue samples were not significant. B: Gene expression levels of Dkk1 in deep infiltrated endometriosis tissue samples was significantly lower than it in not deep infiltrated endometriosis tissue samples

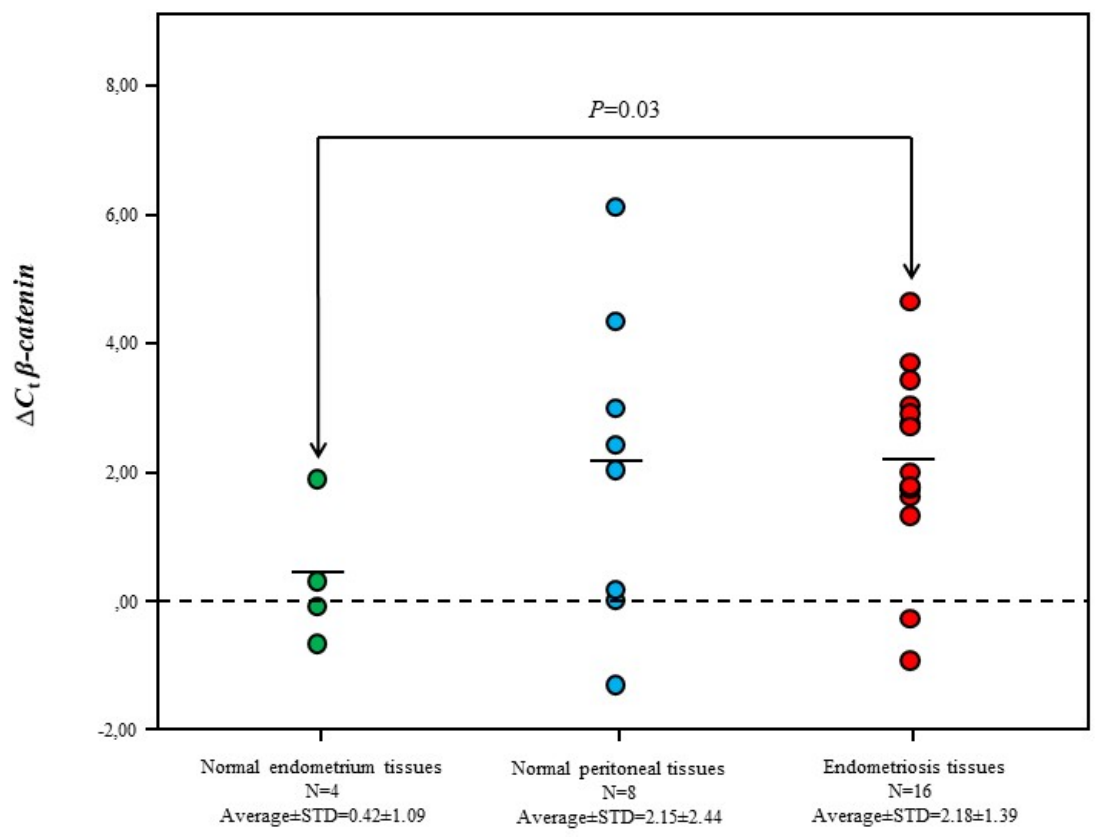

Figure 5. Gene expression levels of $\beta$-catenin in different tissue samples. Gene expression levels of Dkk1 in endometriosis tissue samples was significantly lower than it in normal endometrium tissue samples

In this study, we showed that significant more abundances of Dkk1 protein were determined in serum of endometriosis patients compared with healthy cohorts. However, we found that Dkk1 serum levels in our study cohorts were not affected by disease severity or by disease occurrence. Serum Dkk1 is considered as advantageous biomarker as it has a simple non-invasive detection protocol and is inexpensive to quantify. However, the great variability and non-normal distribution of its levels affected the chances of identifying a clear cut-off value or significant differences related to the clinical features of the groups. Therefore, further large-scale and multicentre studies are absolutely required to confirm our findings introducing serum levels of Dkk1 as a diagnostic biomarker in endometriosis.

We further tested weather Dkk1 expression is dysregulated too at mRNA level. We determined the expression level of $d k k 1$ in 
endometriosis tissues and compared it to its levels either in normal endometrium tissues from women without endometriosis or in normal peritoneal tissues from women with endometriosis. Moreover, we tested $\beta$-catenin expression levels to investigate whether Dkk1is enrolled in endometriosis pathogenicity directly via its function as an inhibitor of the Wnt/ $\beta$-catenin signaling. Our results demonstrated no significant alteration in $d k k 1$ expression levels within the tested tissue groups. However, unlike serum Dkk1 protein concentrations, $d k k 1$ expression levels were increased in endometriosis tissues of patients with deep infiltrated endometriosis compared to them in patients with less disease severity. In addition, there was no significant correlation between serum levels of Dkk1 and $d k k 1$ expression levels in endometriosis tissues from the same patient. These findings suggest that transcript levels by themselves could not be sufficient to predict protein levels in this scenario and to thus explain genotype-phenotype relationship. Moreover, as a secreted glycoprotein, a difference in secondary modifications on it or its ligands could impact its biological properties and functions.

Expression profile of the Wnt/ $\beta$-catenin signaling's components in endometriosis, particularly Dkk1, has been investigated in a few, small, human studies and contradicted results were demonstrated. Findings of Pazhohan and co-workers showed that while Dkk1 geneand protein expression levels in both endometriotic lesions and eutopic endometrium of endometriosis patients were significantly lower than them in the endometrium of healthy subjects, non-phosphorylated $\beta$-catenin (active form of $\beta$-catenin) was more expressed at protein level only [19]. Microarray analysis of eight endometrial biopsies from patients with endometriosis versus seven ones from women without endometriosis identified 91 genes that were significantly upregulated and 115 genes that were significantly downregulated including Dkk1 gene [20]. Brueggmann and colleagues documented that gene expression levels of $d k k 1$ and E-cadherin were downregulated in ovarian endometriosis tissues compared with matching endometrial samples of patients with endometriosis [21]. Data from these studies provided a proof of downregulated expression of $d k k l i n$ endometriosis tissues when compared to non-endometriotic tissues from subjects with- or without endometriosis. On the other hand, it has been showed that $\beta$-catenin mRNA expression levels in endometriosis tissues had no significant cyclical differences compared to eutopic endometrium from women with- or without endometriosis $[19,22]$. However, contradicted observations regarding protein expression of $\beta$-catenin were reported by the last two studies. In a rat model of peritoneal endometriosis, there were no differences in $\beta$-catenin mRNA levels and $\beta$-catenin protein levels in endometriosis samples and their matched endometrium samples of twenty female rats [23].

In our study, although $d k k 1$ expression level in endometriosis tissues was almost the same as it in normal peritoneal tissues of endometriosis patients and lower than it in normal endometrium of subjects without endometriosis, these observations were statistically not significant. Nevertheless, $\beta$-catenin mRNA levels in endometriosis tissues, but not in normal peritoneal samples, were downregulated compared with normal endometrium tissues. These inconsistent observations from our study with the others could be relevant to the variances in cohorts' characteristics such as number of tested cases, cycle phase, location of endometriosis lesions, and disease stages.

In conclusions, the present study demonstrated aberrant expression of certain components of $\mathrm{Wnt} / \beta$-catenin signaling in endometriosis patients represented by decreased $\beta$-catenin at $m R N A$ expression levels and increased Dkk1 at protein expression levels suggesting the enrollment of this transduction pathway in endometriosis pathogenicity. To the best of our knowledge, this is the first study to investigate serum levels of Dkk1 in patients with endometriosis. Hence, Dkk1 could be a useful marker for diagnosing endometriosis. However, this hypothesis needs to be further explored by doing large-scale, multicentre studies to prove the suggested role of Dkk1 as a potential diagnostic protein biomarker for endometriosis.

\section{Acknowledgements}

The authors would like to thank Mrs. Adriana Nistor from the department of Pathology at the University Medical School of Saarland for her assistant doing the histological evaluation of all H\&E sections that were included in this study.

\section{Conflict of interest}

We declare that we have no conflict of interest.

\section{Funding}

This research did not receive any specific grant from any funding agency in the public, commercial or not-for-profit sector.

\section{References}

1. Clevers $\mathrm{H}$ (2006) Wnt/beta-catenin signaling in development and disease. Cell 127 469-480. [Crossref]

2. van der Horst PH, Wang Y, van der Zee M, Burger CW, Blok LJ (2012) Interaction between sex hormones and WNT/b-catenin signal transduction in endome-trial physiology and disease. Mol Cell Endocrinol 358: 176-184.

3. Sanchez AM, Vigano P, Quattrone F, Pagliardini L, Papaleo E, et al. (2014) The Wnt $ß$-catenin signalling pathway and expression of survival promoting genes in luteinized granulosa cells: endometriosis as a paradigm for a dysregulated apoptosis pathway. Fertil Steril 101: 1688-1696.

4. Li J, Dai Y, Zhu H, Jiang Y, Zhang S (2016) Endometriotic mesenchymal stem cells significantly promote fibrogenesis in ovarian endometrioma through the Wnt/ß-catenin pathway by paracrine production of TGF- $\beta 1$ and Wnt1. Hum Reprod 31: 1224-1235.

5. Zhang L, Xiong W, Xiong Y, Liu H, Liu Y (2016) 17 ß-Estradiol promotes vascula endothelial growth factor expression via the Wnt/ß-catenin pathway during the pathogenesis of endometriosis. Mol Hum Reprod 22: 526-535.

6. Semënov MV, Tamai K, Brott BK, Kühl M, Sokol S, et al. (2001) Head inducer Dickkopf-1 is a ligand for Wnt coreceptor LRP6. Curr Biol 11: 951-961. [Crossref]

7. Niehrs C (2006) Function and biological roles of the Dickkopf family of Wnt modulators. Oncogene 25: 7469-7481. [Crossref]

8. Jin H, Wang B, Li J, Xie W, Mao Q, et al. (2015) Anti-DKK1 antibody promotes bone fracture healing through activation of $\beta$-catenin signaling. Bone 71: 63-75.

9. Iyer SP, Beck JT, Stewart AK, Shah J, Kelly KR, et al. (2014) A Phase IB multicentre dose-determination study of BHQ880 in combination with anti-myeloma therapy and zoledronic acid in patients with relapsed or refractory multiple myeloma and prior skeletal-related events. Br J Haematol 167: 366-375. [Crossref]

10. Kasoha M, Bohle RM, Seibold A, Gerlinger C, Juhasz-Böss I, et al. (2018) Dickkopf-1 (Dkk1) protein expression in breast cancer with special reference to bone metastases. Clin Exp Metastasis 35: 763-775. [Crossref]

11. Dong LL, Qu LY, Chu LY, Zhang XH, Liu YH (2014) Serum level of DKK-1 and its prognostic potential in non-small cell lung cancer. Diagn Pathol 9: 10-52.

12. Jiang T, Huang L, Zhang S (2013) DKK-1 in serum as a clinical and prognostic factor in patients with cervical cancer. Int J Biol Markers 28: 221-225.

13. Nnoaham KE, Hummelshoj L, Webster P, d'Hooghe T, de Cicco Nardone F, et al (2011) World Endometriosis Research Foundation Global Study of Women's Health consortium. Impact of endometriosis on quality of life and work productivity: a multicenter study across ten countries. Fertil Steril 96: 366-373.

14. Soliman AM, Yang H, Du EX, Kelley C, Winkel C (2016) The direct and indirect costs associated with endometriosis: a systematic literature review. Hum Reprod 31: 712722 . 
15. American Society for Reproductive Medicine (1997) Revised American Society for Reproductive Medicine classification of endometriosis: 1996. Fertil Steril 67: 817-821. [Crossref]

16. Albertsen HM, Ward K (2017) Genes linked to endometriosis by GWAS are integral to cytoskeleton regulation and suggests that mesothelial barrier homeostasis is a factor in the pathogenesis of endometriosis. Reprod Sci 24: 803-811. [Crossref]

17. Yang YM, Yang WX (2017) Epithelial-to-mesenchymal transition in the development of endometriosis. Oncotarget 8: 41679-41689. [Crossref]

18. Zhang J, Tian XJ, Xing J (2016) Signal transduction pathways of EMT Induced by TGF- $\hat{I}^{2}$, SHH, and WNT and their crosstalks. J Clin Med 5. [Crossref]

19. Pazhohan A, Amidi F, Akbari-Asbagh F, Seyedrezazadeh E, Farzadi L, et al. (2018) The Wnt/B-catenin signaling in endometriosis, the expression of total and active forms of $\beta$-catenin, total and inactive forms of glycogen synthase kinase-3B, WNT7a and DICKKOPF-1. Eur J Obstet Gynecol Reprod Biol 220: 1-5.
20. Kao LC, Germeyer A, Tulac S, Lobo S, Yang JP, et al. (2003) Expression profiling of endometrium from women with endometriosis reveals candidate genes for diseasebased implantation failure and infertility. Endocrinology 144: 2870-2881.

21. Brueggmann D, Jaque JM, Lee AW, Pearce CL, Templeman C (2016) Expression of Wnt signaling pathway genes in human endometriosis tissue - a pilot study. Eur $J$ Obstet Gynecol Reprod Biol 199: 214-215.

22. Matsuzaki S, Darcha C, Maleysson E, Canis M, Mage G (2010) Impaired downregulation of E-cadherin and beta-catenin protein expression in endometrial epithelial cells in the mid-secretory endometrium of infertile patients with endometriosis. $J$ Clin Endocrinol Metab 95: 3437-3445.

23. de Mattos RM, Pereira PR, Barros EG, da Silva JH, Palmero CY, et al. (2016) Aberrant levels of Wnt/ß-catenin pathway components in a rat model of endometriosis. Histol Histopathol 31: 933-942.

Copyright: (2019 Kasoha M. This is an open-access article distributed under the terms of the Creative Commons Attribution License, which permits unrestricted use, distribution, and reproduction in any medium, provided the original author and source are credited. 